Revista de Comunicación y Salud, 2020, Vol. 10, oㅡ 2, pp. 483-502

Editado por Cátedra de Comunicación y Salud

ISSN: 2173-1675

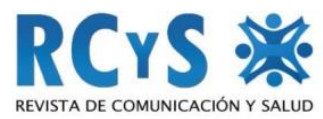

Enviado 15/08/2020

Aprobado 29/09/2020

\title{
LA COMPETENCIA DIGITAL DEL PROFESORADO Y LA ATENCIÓN A LA DIVERSIDAD DURANTE LA COVID-19. ESTUDIO DE CASO
}

\section{Teachers' digital competence and attention to diversity during covid-19: the diversity gap. A case of study}

\author{
Óscar Gómez Jiménez ${ }^{1}$ \\ Universidad de Castilla-La Mancha. España. \\ osquitagomez@gmail.com \\ Javier Rodríguez Torres \\ Universidad de Castilla-La Mancha. España. \\ javier.rtorres@uclm.es \\ Purificación Cruz Cruz \\ Universidad de Castilla-La Mancha. España. \\ purificacion.cruz@uclm.es
}

\section{Resumen}

Tras el establecimiento del estado de alarma provocado por la COVID-19 y, el consiguiente cierre de los centros educativos a nivel nacional, la educación formal se ha trasladado de un contexto de enseñanza presencial a entornos virtuales de aprendizaje en cuestión de horas. La competencia digital supone una asignatura pendiente en la formación del profesorado. A pesar de los esfuerzos puestos por las administraciones educativas en digitalizar el proceso de enseñanza y aprendizaje en los entornos y sistemas formales educativos, su aplicación en las rutinas cotidianas de enseñanza dista mucho de la realidad. Además, es necesario tener en cuenta la diversidad del alumnado y aplicar medidas de disminución de las desigualdades del alumnado, ya que el rol compensador de la escuela (como institución) no puede suplirse a través de la virtualización de la enseñanza.

A través de este estudio que presentamos, vamos a analizar la competencia digital de los docentes y las medidas de atención a la diversidad durante la situación de cierre de los centros educativos por la COVID-19, a través de un estudio de caso del centro educativo, de titularidad religiosa, "Colegio Sagrado Corazón de Jesús y María

1 Óscar Gómez Jiménez: Profesor en el Colegio Sagrado Corazón de Jesús y María Inmaculada (Miajadas, Cáceres) y Doctorando en Investigación en Artes, Humanidades y Educación por la Universidad de Castilla-La Mancha. Especialista en atención a la diversidad y legislación educativa. 
La competencia digital del profesorado y la atención a la diversidad durante la COVID-19

Inmaculada" de Miajadas (Cáceres). Este estudio nos ha permitido definir los niveles de competencia digital del profesorado durante el confinamiento, pero también ha puesto de manifiesto, el deficiente tratamiento específico e individualizado de los alumnos con necesidades educativas especiales de apoyo o refuerzo educativo.

Palabras clave: COVID-19, Estudio de caso, Alfabetización digital, Enseñanza a distancia, Atención a la diversidad.

\section{Abstract}

Following the establishment of the state of alarm caused by COVID-19 and the consequent closure of educational centers nationwide, formal education has moved from a face-to-face teaching context to virtual learning environments in a matter of hours. Digital competence is a pending subject in teacher training. Despite the efforts made by educational administrations to digitize the teaching and learning process in formal educational environments and systems, its application in daily teaching routines is far from reality. Furthermore, it is necessary to take into account the diversity of the students and apply measures to reduce the inequalities of the students, since the compensatory role of the school (as an institution) cannot be supplied through the virtualization of teaching.

Through this study that we present, we are going to analyze the digital competence of teachers and the measures of attention to diversity during the closure of schools due to COVID-19, through a case study of the educational center, of religious ownership, "Colegio Sagrado Corazón de Jesús y María Inmaculada" of Miajadas (Cáceres). This study has allowed us to define the levels of digital competence of teachers during confinement, but it has also revealed, in this specific study, the deficient specific and individualized treatment of students with special educational needs for support or educational reinforcement.

Keywords: COVID-19, Case of study, Digital literacy, Distance learning, Attention to diversity.

\section{Cómo citar el artículo}

Gómez Jiménez, O., Rodríguez Torres, J. y Cruz Cruz. P. (2020). La competencia digital del profesorado y la atención a la diversidad durante la COVID-19. Estudio de caso. Revista de Comunicación y Salud, 10 (2), 483-502. doi: https://doi.org/10.35669/rcys.2020.10(2).483-502

\section{INTRODUCCIÓN}

La competencia digital se refiere a las habilidades, destrezas y conocimientos que disponen los docentes para llevar a cabo un proceso formativo, empleando la tecnología desde una perspectiva crítica, segura y pedagógica (Pozo, López, Fernández y López, 2020). 
La competencia digital del profesorado y la atención a la diversidad durante la COVID-19

La competencia digital supone una asignatura pendiente en la formación del profesorado. A pesar de los esfuerzos puestos por las administraciones educativas en digitalizar el proceso de enseñanza y aprendizaje en los entornos y sistemas formales educativos, su aplicación en las rutinas cotidianas de enseñanza dista mucho de la realidad. Los planes de educación superior y las formaciones permanentes del profesorado en materia de enseñanza virtual hacen gran hincapié en la digitalización de la enseñanza y el uso de las TIC de manera ordinaria y continuada dentro del sistema educativo, en todos sus niveles.

La situación educativa española se encuentra en un momento excepcional, debido a la crisis internacional causada por la COVID-19. El 13 de marzo de 2020 se anunciaba el cierre de los centros educativos del territorio nacional y el comienzo del confinamiento de la población española en sus hogares (BOE, 2020a; EFE, 2020). Los centros se encuentran en una situación sobrevenida, para la que no están preparados: la virtualización de la enseñanza, por una duración (aunque principalmente se estableció por quince días) indeterminada.

En ese momento, las administraciones educativas (tanto regionales como el Ministerio de Educación y Formación Profesional) comienzan una carrera a contrarreloj para establecer las normas de funcionamiento de los centros durante el confinamiento de la población escolar española, así como el seguimiento de las clases a través de los medios y dispositivos de los centros educativos (BOE, 2020b). Los docentes se ven obligados a cambiar las aulas por sus espacios domésticos, donde seguir impartiendo los contenidos en un segundo trimestre prácticamente finalizado (cuyas evaluaciones se encontraban completadas) y con la esperanza de retomar la presencialidad después del periodo festivo de Semana Santa.

Un despliegue de plataformas (Juste, 2020) pusieron sus recursos disponibles para la comunidad educativa (de manera gratuita en la mayoría de los casos) y los docentes tuvieron que desplegar sus conocimientos digitales para una transformación educativa de la que no se estaba preparado, a pesar del intento de transformación digital de la gran mayoría de los centros, que se encontraba comenzada, en fases iniciales o medias, o por comenzar.

Docentes y familias aprendieron o intentaron aprender a digitalizar su concepto sobre la educación y se empezaron a establecer canales y plataformas donde los alumnos pudieran acceder al seguimiento de sus clases, entrega de trabajos y comunicación directa con los profesores. Todo ello, sumado a la disposición de recursos tecnológicos personales del profesorado (Cueto, 2020) y de las familias, lo cual puede llegar a suponer el establecimiento de una nueva categorización del alumnado en función de sus recursos de acceso y seguimiento educativo, durante el confinamiento y desescalada de la crisis social y educativa provocada por el SARS-CoV-2.

Es necesario hablar de dos brechas digitales, una producida por la falta de recursos tic en las familias y otra provocada por la falta de atención digital individualizada según

Revista de Comunicación y Salud, 2020, Vol. 10, nº 2, pp. 483-502 
las características personales del alumnado y la sobrecarga laboral del docente. Conllevando una disminución de la función compensadora que permita que el alumnado se encuentre en igualdad de condiciones materiales y herramientas culturales para enfrentarse al proceso de enseñanza y aprendizaje, a la misma vez que se le acompañe durante el proceso educativo, proporcione estabilidad emocional o dote de recursos necesarios para aprender (Díez y Gajardo, 2020).

Esta nueva situación educativa, destacada por opiniones diversas sobre el modelo educativo y la digitalización de la enseñanza de una manera apresurada y sin la debida formación por parte de los profesionales y la comunidad educativa (Martin y Rogero, 2020), centra los esfuerzos en la continuidad educativa del alumnado, mientras que son evaluadas y trabajadas las competencias digitales del profesorado bajo el marco de la situación sanitaria y social establecida en el país y a nivel mundial.

Como hemos indicado anteriormente, la situación excepcional de enseñanza requiere del desarrollo y puesta en práctica de los conocimientos relativos a las aplicaciones didácticas en los entornos digitales adquiridas por los docentes y la creación de didácticas específicas para cada uno de los discentes. Para ello, a través de este estudio que presentamos, se analiza la competencia digital de los docentes a través de un estudio de caso del centro educativo, de titularidad religiosa, "Colegio Sagrado Corazón de Jesús y María Inmaculada" de Miajadas (Cáceres) y la personalización de dichos recursos en alumnos con necesidades educativas especiales.

\subsection{Marco Contextual.}

Las Tecnologías de la Información y la Comunicación (TIC) son recursos que contribuyen significativamente a la innovación y el planteamiento de nuevos procesos culturales y educacionales. Las políticas educativas, desde este paradigma, se han enfocado a la implementación de programas y recursos que faciliten la incorporación de las TIC en todos los niveles del sistema educativo para alfabetizar digitalmente al alumnado, dando lugar a grandes inversiones en equipamiento e infraestructuras, dentro de los centros educativos, así como la creación de planes formativos para el profesorado o la creación de repositorios y direcciones de materiales digitales (Roig y Flores, 2014).

Este proceso de integración de las TIC dentro de los centros educativos ha sido mucho más lento de lo esperado (si lo comparamos con otras administraciones o instituciones sociales), ya que hay tres elementos para tener en cuenta en las dificultades de digitalización: el entorno social, las infraestructuras y el profesorado. Respecto a este último, es necesario incidir en las competencias digitales que los docentes cuentan y en cómo estas se desarrollan dentro de los entornos educativos.

El Instituto Nacional de Tecnologías Educativas y Formación del Profesorado, España, (INTEF) establece un Marco Común de Competencia Digital Docente dividido en 6 niveles, 21 competencias y 5 áreas que desarrollan la competencia digital como 
una competencia clave que cualquier persona debe haber desarrollado para poder incorporarse a la vida adulta de manera significativa (Moreno, Gabarda y Rodríguez, 2018). Para su desarrollo, a nivel nacional se han establecido diferentes planes de impulso de las Tecnologías de la Información y la Comunicación (en adelante, TIC), como el Marco Estratégico de Desarrollo Profesional Docente, el Plan de Cultura Digital en la Escuela o el Proyecto DIGCOMP de la Unión Europea; siendo todos estos planes y marcos estratégicos un espacio de referencia para el impulso del manejo de las TIC dentro del entorno educativo.

Si nos movemos a un plano más normativo en educación, la Ley Orgánica 2/2006, de 3 de mayo de Educación (BOE, 2006), modificada por el artículo único de la Ley Orgánica 8/2013, de 9 de diciembre, para la Mejora de la Calidad Educativa (BOE, 2013) expone, en su artículo 111 bis, la necesidad de trabajar las TIC de forma transversal y complementaria, así como ha de ser una competencia clave a trabajar a lo largo de la escolarización del alumnado. Para ello, y siguiendo lo establecido en las recomendaciones de la Unión Europea (Valle y Manso, 2013), se ha realizado un gran esfuerzo nacional para la implementación de la tecnología educativa dentro de los centros, así como la incorporación de esta dentro de los proyectos educativos para dar cumplimiento a la competencia digital de manera eficaz.

Siguiendo lo expuesto por Gabarda, Rodríguez y Moreno (2017), encontramos una evolución en el desarrollo de la aplicación de la tecnología en el ámbito educativo, donde se destaca el énfasis en el establecimiento de programas de refuerzo para el aprendizaje de las TIC para el profesorado. Programas de formación permanente, como los creados por INTEF o por los Centro Regionales de Formación del Profesorado, ofrecen la oportunidad de actualización de las competencias digitales al profesorado, con el objetivo de realizar un desempeño efectivo basado en la movilización de recursos tecnológicos, informacionales, axiológicos, pedagógicos y comunicativos (Marqués, 2008).

Fuentes y López (2018) exponen que cada individuo social se ve involucrado, obligatoriamente, a incorporarse a la alfabetización digital para poder desempeñarse con soltura dentro de la era digital. Esto supone una afirmación esencial dentro del entorno educativo, ya que es innegable la inclusión de las TIC en los espacios educativos ya que, en la búsqueda de la calidad y excelencia educativa, es necesario la optimización del proceso de enseñanza y aprendizaje del alumno y la implementación de recursos pedagógicos variados, siendo aquellos de índole tecnológica los imprescindibles a utilizar para alcanzar tal fin.

Además, la situación actual sobrevenida nos hace replantear el momento en el que se encuentra la atención a la diversidad del alumnado y qué medidas se han de tomar, ya que encontramos en las TIC un recurso de recursos que nos pueden ayudar a atender a la diversidad de necesidades individuales de nuestro alumnado, tanto en el centro educativo como en una situación excepcional de aprendizaje a distancia. 
La construcción del nuevo escenario educativo, en el que la comunidad educativa se encuentra inmerso, depende de la autonomía de los centros y de la alfabetización mediático-informacional de las familias (Trujillo, 2020). Se ha tener en cuenta factores como: el acceso a Internet en el hogar familiar, la competencia de las familias (tanto digital como cultural) y la situación personal y familiar del alumnado. Las familias con más necesidades carecen de condiciones materiales, culturales, pedagógicas, formativas e, incluso, emocionales para poder enfrentar la situación personal y familiar derivada de una paralización social y económica.

Díez y Gajardo (2020) indican que la brecha social y socioeconómica que sufre gran parte de la población se ve potenciada ante una situación de crisis y de confinamiento. A esta brecha social se suma la brecha digital y tecnológica, aumentando más aún la desigualdad. A pesar de los esfuerzos en la dotación de recursos tecnológicos (por parte de las Administraciones Educativas y los centros escolares), la analfabetización digital hace necesario replantear los procesos de enseñanza y aprendizaje actuales, en pro de la búsqueda de metodologías que favorezcan el aprendizaje y tengan en cuenta el grado de autonomía y desarrollo del alumnado (Moreno, 2020).

El impacto de la situación educativa actual derivado de la emergencia social por la COVID-19, va a tener un efecto desigual entre el alumnado, ya que los apoyos académicos, los recursos tecnológicos, las habilidades no cognitivas y el nivel cultural supone un hándicap para los alumnos más desfavorecidos que resulta difícil de suplir a través de la formación virtual sin una formación digital avanzada por parte del profesorado. Sumando las dificultades que algunos de nuestros alumnos, por sus características especiales, tienen para seguir el ritmo de la clase, o seguir su plan de trabajo individualizado sin la presencialidad de su tutor o maestro especialista, contando sólo con los recursos tecnológicos como único medio de comunicación.

\subsection{Caracterización del centro educativo}

El centro educativo "Colegio Sagrado Corazón de Jesús y María Inmaculada" situado en Miajadas (Cáceres), pertenece a la Congregación Religiosa "Hijas de la Virgen de los Dolores" y fue fundado en la década de los años 20 , en su afán por llevar la educación y la cultura a una tierra y a unas gentes sumidas en la pobreza y, aunque la realidad social, económica y política de hoy resulta muy diferente, la filosofía pedagógica del centro sigue siendo la misma, poniendo como centro de su acción pedagógica, la atención a los colectivos en riesgo de inclusión de la localidad. El centro educativo cuenta con 487 alumnos y 46 profesores.

Respecto a la línea pedagógica del centro, cuenta con diversos premios nacionales e internaciones en busca de la calidad y eficiencia en el proceso educativo, implicando, no solo a la comunidad de profesores y alumnos, sino también a los padres, en un proceso de formación y actualización pedagógica permanente.

El peculiar entorno miajadeño arroja los siguientes datos:

Revista de Comunicación y Salud, 2020, Vol. 10, nº 2, pp. 483-502 
La competencia digital del profesorado y la atención a la diversidad durante la COVID-19

1. La tasa de población infantil y juvenil es superior a la media provincial, por lo que el número de alumnos en edad escolar llega casi a las 2000 personas en todo el municipio.

2. El incremento poblacional es constante en los últimos años (con ligero decrecimiento en 2011)

3. Se observa una tasa homogénea de nacimientos (en torno a 90-100 niños por año). Esta tasa de nacimientos hace necesario mantener las unidades concertadas con nuestro centro al no poder cubrir la Enseñanza Pública el total de plazas demandadas.

4. En la población existe una amplia demanda de nuestro centro por parte de los padres.

5. Miajadas cuenta con un amplio espectro de población inmigrante (superior al $18 \%$ ) y otras minorías como gitanos (en torno al $5 \%$ de la población) que también demandan este tipo de educación para sus hijos.

6. A nivel económico, la crisis se ha cebado especialmente con el sector de la construcción, experimentándose la caída de algunas empresas importantes y un incremento del paro notable en este sector. Los trabajadores autónomos son el verdadero motor económico de Miaja-das, aunque todos los sectores están directa o indirectamente relacionados con la agricultura.

Respecto a los niveles educativos de los que cuenta el centro educativo, el centro cuenta con las etapas de Educación Infantil, Educación Primaria y Educación Secundaria Obligatoria (de manera gratuitas para el alumnado) y con Bachillerato (etapa que es concertada para las familias). El centro se enmarca en dos líneas (es decir, dos clases por curso) y cuenta con sección bilingüe en la etapa de Educación Primaria y Educación Secundaria Obligatoria. El centro cuenta con dos grupos diferenciados de alumnado con necesidades educativas especiales: alumnos de compensación educativa (donde se incluyen a aquellos procedentes de minorías y etnias, que suponen un total de 32 alumnos) y alumnos con necesidades específicas de apoyo educativo (que suponen un total de 11 alumnos, de los cuales 4 presentan Trastorno por Déficit de Atención con Hiperactividad y 9 presentan necesidades educativas especiales).

\section{OBJETIVOS}

Este estudio de caso tiene como objetivo principal conocer la autopercepción de la competencia digital del profesorado y su preparación previa para el trabajo en entornos digitalizados y compartidos con el alumnado en el Centro Educativo "Sagrado Corazón de Jesús y María Inmaculada". Para alcanzar el objetivo principal, hemos planteado los siguientes objetivos:

1. Establecer el nivel de competencia digital del profesorado antes y después del cierre de los colegios por la situación de emergencia sanitaria provocada por la COVID-19.

2. Conocer el nivel de formación del profesorado en el uso de las TIC y de los recursos tecnológicos de los que dispone el centro

Revista de Comunicación y Salud, 2020, Vol. 10, nº 2, pp. 483-502 
La competencia digital del profesorado y la atención a la diversidad durante la COVID-19

3. Determinar el uso de los recursos TIC, en época de pandemia, según las características individuales del alumnado

\section{METODOLOGÍA}

En la actualidad, existen diferentes métodos de investigación, pero "la investigación cualitativa ha alcanzado un alto grado de desarrollo, contundencia metodológica y científica que no es exclusivo de la investigación cuantitativa" (Erazo 2011; citado en Hernando y Zwerg-Villegas, 2012). Para el siguiente trabajo, hemos considerado oportuno utilizar la investigación cualitativa pues, como afirma Flick (2012) utilizar este tipo de métodos puede ayudarnos a describir las distintas perspectivas y significados subjetivo y social de los participantes, así como analizar las interacciones e interrelaciones que se dan entre éstos y su entorno. Y más concretamente el estudio de caso, ya que es considerado un proceso de indagación sistemático y en profundidad de entidades educativas únicas (Bisquerra, 2009), que describe una realidad muy particular y los procesos que en dicho contexto se desarrollan, para así intentar comprender una realidad de lo más diversa y heterogénea. La anterior reflexión nos permite interpretar los resultados, a partir de la valoración y los significados de los actores consultados, sobre el nivel de competencia digital docente y su aplicación durante la situación de emergencia sanitaria por la COVID-19 en los entornos educativos.

Como ya hemos indicado anteriormente, el contexto del estudio corresponde a un centro de titularidad privada-concertada ubicado en la localidad de Miajadas (Cáceres). Para la realización del estudio se ha contado con la totalidad del claustro de profesores, es decir, la muestra seleccionada la conformaron 46 docentes que engloban todas las etapas educativas impartidas en el centro. Del $100 \%$ de personas seleccionadas, el $34,7 \%$ corresponde a hombres y un $65,3 \%$ a mujeres. La media de edad es de 45,8 años y la de años de experiencia docente es de 13,2 años. Se seleccionaron todos los profesores porque el centro reunía unas características bien diferenciadas: incluye a todas las etapas educativas obligatorias, tiene una trayectoria y experiencia en el tratamiento a la diversidad y todos los docentes, de una forma u otra, están implicados en las dinámicas de inclusión avaladas por la formación continuada. Además, al tratarse de un colegio concertado, la movilidad del profesorado es muy leve.

La recogida de información se realizó a través de una entrevista semiestructurada, dirigida a cada miembro del claustro docente y con una duración aproximada de 30 minutos. El cuestionario cuenta con un total de 20 preguntas, de las cuales 8 son cerradas y 12 abiertas. Las preguntas cerradas recogen la información personal y profesional del profesorado (sexo, años de experiencia docente o etapa educativa en la que imparten docencia, entre otras) y las preguntas abiertas recogen la información sobre el nivel de competencia digital del profesorado, la formación docente previa en el uso de las TIC y el uso de estas para la atención individualizada del alumnado durante el cierre del centro. Para ello, las preguntas abiertas se dividieron en tres principales grupos (haciendo referencia a los objetivos de este estudio). El primer grupo de preguntas, sobre el nivel de competencia digital del profesorado, cuenta con preguntas 
La competencia digital del profesorado y la atención a la diversidad durante la COVID-19

como "Describa su formación previa en el uso de las TIC", ¿Qué herramientas o plataformas digitales utiliza en sus clases presenciales" o "¿Qué formación TIC ha recibido durante el cierre del centro? ¿Ha aplicado lo aprendido a sus clases online?". El segundo grupo de preguntas, sobre la formación del profesorado en el uso de las TIC, cuenta con preguntas como " ¿Cuenta el centro educativo con recursos tecnológicos suficientes para la integración de las TIC en el aula? ¿En qué aspectos debería mejorar?" o “¿Cómo considera que ha sido la integración de las TIC en la dinámica general del centro durante el cierre de este? ¿Se ha hecho un uso correcto en su integración y formación a la comunidad educativa?". El tercer grupo de preguntas, sobre el uso de las TIC para la atención individualizada del alumnado general y del alumnado con necesidades educativas especiales, cuenta con preguntas como " ¿Considera que se ha atendido a las necesidades individuales del alumnado durante el cierre del centro educativo? ¿Se han utilizado las TIC convenientemente para llegar a tal objetivo?”, “¿Qué dificultades cree usted que se han encontrado para atender al alumnado durante este periodo de tiempo? ¿Cree que un mejor uso de las TIC podría favorecer mejorar la atención educativa del alumnado con necesidades especiales de aprendizaje? ¿De qué manera se podría realizar?”. El cuestionario ofreció mucha información, pero solamente se escogió la información referida sobre el objeto de la investigación.

El equipo directivo del centro creyó conveniente que todos los docentes participaran en la encuesta para poder incluir los resultados en la memoria del curso y en las propuestas de mejora solicitadas por la Administración Educativa Regional de Extremadura. Se utilizó la plataforma Teams (cedida por la Universidad de Castilla la Mancha) y se grabaron cada una de las videoconferencias. Una vez concluidas las grabaciones, fueron visionadas por los tres investigadores de forma individual, que previamente habían establecido las siguientes categorías semánticas: Percepción sobre el uso de las TIC - Necesidades formativas para la mejora de la competencia digital docente - Dificultad del uso de las TIC en situaciones de aprendizaje extraordinarias.

Las respuestas fueron transcritas respetando el discurso de los actores. Se efectuó un análisis comparativo sistemático de los registros que permitió delimitar las dimensiones que demarcaron el análisis, extrayendo las etiquetas utilizadas para la investigación y que más se repetían en las entrevistas y convirtiendo, a través de la triangulación, los resultados unificados en categorías que permitieron establecer el nivel de competencia digital según los descriptores de los niveles expuestos por el Instituto Nacional de Tecnologías Educativas y Formación del Profesorado y su didáctica a cada uno de los sujetos de estudio. Esto llevó a: a) codificar toda la información; b) agrupar datos cuantitativos en porcentajes y frecuencias; c) realizar tablas para sintetizar la información; d) interpretar los datos en el contexto en que fueron recogidos; e) Aplicar el test de Fisher para contrastar si las variables cualitativas estaban relacionadas mediante test de frecuencia y por tanto contábamos con fuerza de asociación; f) extraer conclusiones; y d) compartir dichas conclusiones con el centro objeto de estudio. 
La competencia digital del profesorado y la atención a la diversidad durante la COVID-19

\section{RESULTADOS}

Uno de los aspectos importantes de este estudio de caso es el uso de las TIC dentro del entorno educativo, por parte del profesorado. Para ello, se establecieron dos momentos en la utilización de los recursos: el uso de las TIC de manera generalizada durante cuando los alumnos asistían a clase de manera presencial, antes de la pandemia y la aplicación de los recursos generales durante la suspensión presencial de las clases, realizando actividades sincrónicas y anacrónicas (videoconferencias, tutorías, envío de materiales, etc.).

Durante la presencialidad de la enseñanza en los centros educativos, los resultados de las entrevistas realizadas nos muestran que el uso de las TIC en el centro es de soporte a los modelos pedagógicos del profesorado. Su principal uso es para la utilización del libro digital o la proyección de videos complementarios a la explicación pertinente. La pizarra digital interactiva (PDI) se utiliza como proyector de videos y animaciones y el uso de herramientas especializadas se limita a su uso en momentos concretos y por un corto periodo de tiempo (por ejemplo, como herramienta de evaluación de una sesión de aprendizaje). El profesorado muestra un bajo nivel de integración de sus competencias digitales dentro de la dinámica general del aula, ya que no destaca una creación de recursos o una integración completa dentro de la programación didáctica, es decir, se utiliza como recurso complementario, primando los recursos tradicionales, como el libro o la pizarra de tiza. Por parte del centro, se potencia la utilización de las tecnologías, pero la utilización va ligada a experiencias piloto (cuya duración ha sido interrumpida por el cierre del centro).

Si nos movemos a la utilización de las TIC durante el periodo de virtualización de la enseñanza, encontramos un aumento de las competencias digitales de los docentes, motivado por la necesidad de trasladar el proceso de enseñanza y aprendizaje a un entorno virtual de manera indeterminada. Los docentes manifiestan un aumento en la utilización de recursos tecnológicos y de entornos virtuales de enseñanza. Se ha potenciado la utilización de plataformas de comunicación (a criterio de los conocimientos de cada profesor respecto a qué plataforma utilizar) y se ha potenciado la presencia del centro en los entornos digitales (a través de la creación de páginas webs, dominios personalizados y entornos de comunicación comunes para el profesorado y el alumnado). Esto ha favorecido el interés por un correcto uso de las herramientas digitales, por parte del profesorado, a través de formaciones internas (tanto a nivel centro como por parte de las administraciones educativas), adaptado al nivel competencial de cada profesor y con el objetivo último de lograr una extrapolación a sus necesidades docentes y de aula.

A partir de los datos obtenidos en las entrevistas a los docentes del centro educativo, encontramos que el profesorado tiene una formación previa baja en el uso de las TIC, ya que la integración de estas en las sesiones de enseñanza y aprendizaje es mínima o de soporte a las estrategias expositivas docentes, correspondiéndose esta formación básica en un nivel A1/A2 (INTEF, 2017). Tras analizar los resultados de las preguntas sobre la mejora en el uso de las TIC durante el cierre del centro educativo, cabe

Revista de Comunicación y Salud, 2020, Vol. 10, № 2, pp. 483-502 
destacar una mejora de la competencia digital de los docentes (habilidades, destrezas y conocimientos que disponen para llevar a cabo un proceso formativo, empleando la tecnología) tras la situación de confinamiento, situándose en un nivel competencial de manejo de nivel A2/B1 (INTEF, 2017). Si nos centramos en las áreas que componen la Competencia Digital Docente, los resultados por área serían los siguientes:

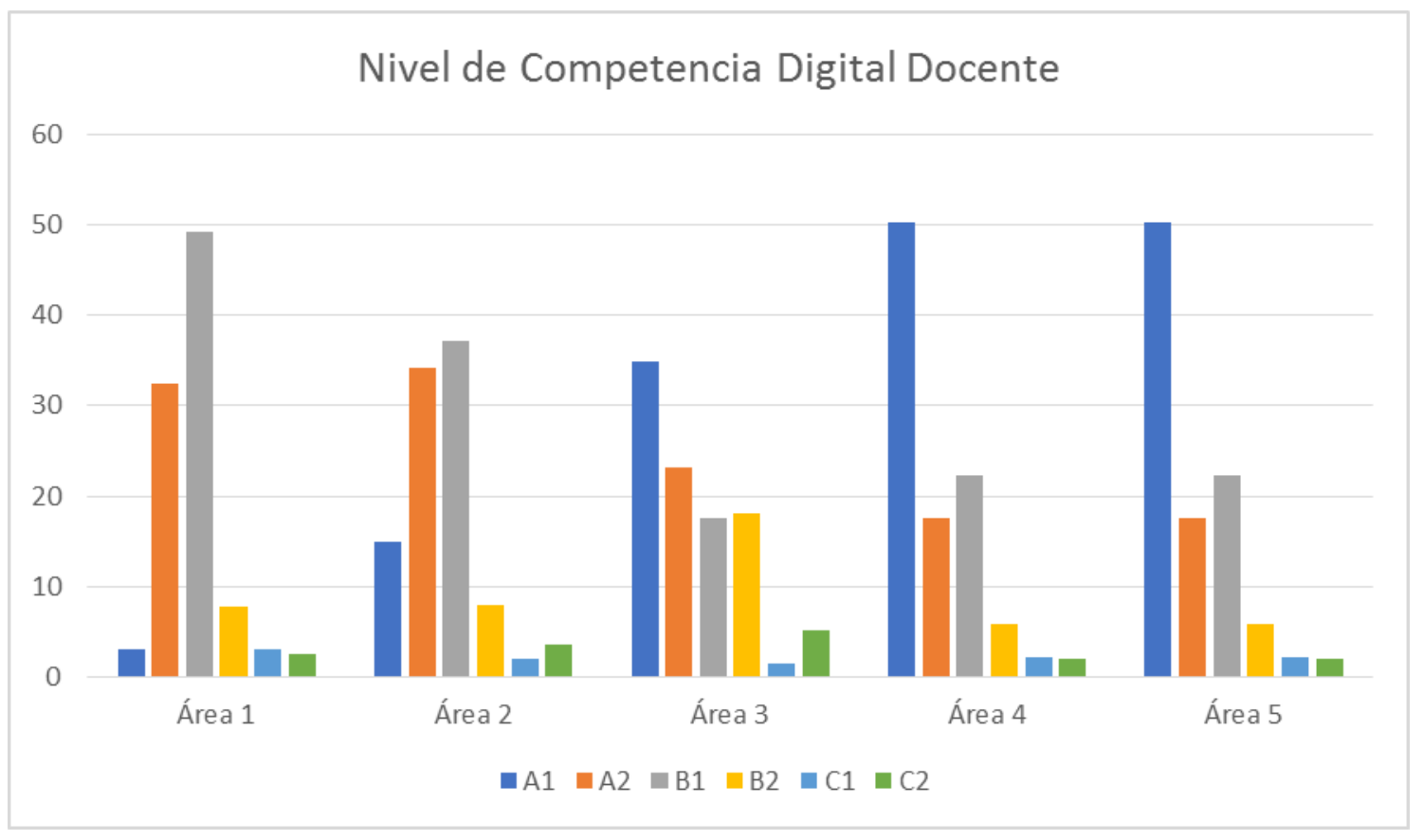

Gráfico 1. Nivel de Competencia Digital Docente.

Fuente: Elaboración propia.

Analizados los datos que presentamos en el gráfico anterior, encontramos que el nivel de la Competencia Digital Docente se encuentra en torno a un nivel A2/B1. Siguiendo los descriptores de los niveles expuestos por el Instituto Nacional de Tecnologías Educativas y Formación del Profesorado (INTEF, 2017), encontramos que el profesorado posee un nivel de competencia básico o intermedio, con cierto nivel de autonomía en la resolución de problemas sencillos y con necesidad de ayuda puntual y apoyo apropiado para la resolución de problemas, pudiendo desarrollar su competencia digital de manera apropiada.

Encontramos un profesorado con un nivel usuario de competencia digital, el cual utiliza las Tecnologías de la Información y la Comunicación para cuestiones cotidianas y de comunicación directa (correo electrónico, plataforma de gestión docente, etcétera), pero que no cuenta con las estrategias adecuadas para un proceso de virtualización de la enseñanza en un entorno de excepcionalidad educativo. Los resultados de las entrevistas trasladan una deficiente formación TIC por parte del profesorado, a pesar de las oportunidades formativas ofrecidas por el Centro de Formación del Profesorado de Extremadura y por el propio centro educativo. 
La competencia digital del profesorado y la atención a la diversidad durante la COVID-19

Por áreas concretas de la Competencia Digital Docente, obtenemos los siguientes datos:

1. Área 1. Información y alfabetización informacional: El profesorado presenta un nivel óptimo en el uso de los aspectos básicos de manejo del ordenador y del uso de los recursos digitales (destacando el uso de los recursos TIC a nivel usuario), así como utiliza espacios de almacenamiento y recuperación de información, datos y contenidos digitales para uso personal y profesional.

2. Área 2. Comunicación y colaboración. En esta área, el desarrollo de la competencia digital está enmarcado en el uso personal que se hace de la tecnología para compartir e interaccionar, destacando el uso de plataformas y espacios virtuales de uso frecuente docente (plataforma Rayuela, YouTube, Servicios de mensajería, etcétera). Respecto a la gestión de la identidad digital y la netiqueta, el nivel competencia es menor que el computo global del área.

3. Área 3. Creación de contenidos digitales. Los resultados del nivel competencia en esta área son menores que en las dos áreas anteriores. La creación de contenidos digitales y su marco legal (derechos de autor, licencias, permisos, programación, etcétera) es limitado, ya que la formación en estos aspectos se limita al uso de determinadas plataformas enfocadas a momentos concretos de aula (evaluación y creación de presentaciones), aunque se ha visto incrementado el trabajo en el área durante el periodo de enseñanza virtual por la COVID-19.

4. Área 4. Seguridad. El equipo docente presenta un nivel muy bajo de adquisición en esta área, ya que los conocimientos sobre la protección de equipos y dispositivos se limita a un uso de nivel usuario, presentado grandes carencias en la protección de datos personales e identidad digital.

5. Área 5. Resolución de problemas. La resolución de problemas derivados del uso de las tecnologías en el aula (y a nivel personal), es muy limitado, ya que el uso de los dispositivos y de los programas o plataformas es rutinario y se cuenta, en el centro, con un área o departamento encargado de la identificación y resolución de estos problemas, a disposición del profesorado.

Esta apreciación del nivel competencial del profesorado por áreas queda matizada por un ligero incremento en las habilidades y destrezas digitales. El cierre del centro educativo ha puesto en valor las capacidades y los conocimientos tecnológicos del profesorado, siendo incrementado el uso de las TIC durante este periodo de tiempo.

Siguiendo esta apreciación, podemos determinar que el nivel competencias digital de los docentes del centro educativo a estudio, se sitúa más cerca de un nivel intermedio, ya que la situación sobrevenida ha resultado positiva para la mejora de la competencia profesional.

Referente al objetivo dos planteado en el estudio, la formación inicial del profesorado con respecto a las TIC no es suficiente para un correcto desarrollo competencial y su aplicación directa dentro de la dinámica de aula. Además de este aspecto destacable (donde tenemos que resaltar que nos encontramos con un profesorado con una

Revista de Comunicación y Salud, 2020, Vol. 10, nº 2, pp. 483-502 
La competencia digital del profesorado y la atención a la diversidad durante la COVID-19

experiencia docente media de 13,2 años), es necesario remarcar que el centro no contaba con los recursos necesarios por aula y niños para poder hacer una aplicación completa de las TIC dentro de las sesiones didácticas y por lo tanto desarrollar la competencia digital de la comunidad educativa.

La opinión generalizada del profesorado es que no se cuenta con unos recursos tecnológicos actualizado en todas las aulas, además de los problemas técnicos (de conectividad y de actualización de los recursos) que pueden presentar durante el curso escolar. Por parte del centro, se empieza a contar con un plan de actualización de recursos y de digitalización del entorno educativo, que de forma urgente se ha visto incrementado en su tiempo de aplicación debido al cierre del centro por la COVID-19 (a través de dotación de cuentas de correo electrónico al profesorado, formación en el paquete de aplicaciones de G-Suite, utilización de plataformas de comunicación con el profesorado y familias, etcétera).

La motivación hacia la mejora de las competencias digitales individuales del profesorado se ha visto aumentada durante este periodo de tiempo, creciendo el uso de aplicaciones específicas, mencionadas anteriormente, que complementan el proceso de enseñanza, así como la acreditación en el uso de plataformas educativas o la solicitud de formación especializada en determinados campos de las tecnologías educativas.

La investigación indica que la utilización de las TIC ha experimentado un aumento conceptual y procedimental en la programación docente de los profesores en esta época de pandemia. Estando integradas en las sesiones virtuales y haciendo de ellas un uso casi diario. Es digno de apreciar como el alumnado, en líneas generales y sin olvidarnos de la brecha digital apreciada en algunas familias, se ha adaptado rápidamente a los cambios tecnológicos, un hecho que se evidencia en el contacto directo que han tenido a lo largo de las clases on-line.

Finalmente, tras el estudio de caso del centro educativo, el nivel de competencia digital docente queda condicionado de diversos factores como la edad, la formación inicial del profesorado y la disposición de recursos del aula. En el caso concreto de este centro, hemos podido comprobar como el nivel competencial ha mejorado (de un nivel básico a un nivel usuario) durante el confinamiento y el periodo de enseñanza online. Esta mejora no debería de ser algo aislado, sino una constante que ayude al plan de digitalización del centro y al propio desarrollo personal y profesional del profesorado, ya que la mejora de las competencias profesionales recae en una mejora educativa a corto y medio plazo.

En relación al tercer objetivo planteado, se ha apreciado que, en gran medida, no se ha trabajado de forma intensa con los alumnos con Necesidades Educativas Especiales (NEE), los cuales muestran dificultades mayores a las del resto de sus compañeros para desarrollar los aprendizajes esperados a su nivel y que requieren de un apoyo especializado, pero que sí deberían gozar, al igual que el resto, de una variedad de recursos tecnológicos en el ámbito educativo acordes a su nivel curricular y que les 
La competencia digital del profesorado y la atención a la diversidad durante la COVID-19

ofrezca la oportunidad de un proceso de enseñanza-aprendizaje innovador, motivador e individualizado.

Como se ha podido comprobar en sus respuestas, son numerosos los obstáculos o dificultades que han impedido este desarrollo curricular individualizado. Entre ellos podemos destacar: superados por la situación, novedad de un entorno absolutamente desconocido, sobrecarga de trabajo con el grupo ordinario, ratios muy altas, falta de recursos, diversidad de situaciones familiares y necesidad, en algunos de los casos, de la ayuda de un adulto del entorno familiar, reorganización de los elementos programáticos y adaptaciones curriculares significativas, falta de formación y poca confianza en que el uso de los recursos TIC funcionaran con este tipo de alumnado sin la presencialidad del tutor.

Todos estos aspectos llevaron a una actuación más generaliza del grupo de alumnos, aportando los planes de trabajo individuales para aquellos alumnos ACNEAES, pero sin poder hacer más en cuanto a metodología individualizada en la utilización de las TIC.

\section{CONCLUSIONES}

La incorporación de las TIC interrelacionando contenidos, pedagogías y tecnologías ha de darse a través de la mejora digital de los docentes. Como hemos visto en el estudio de caso, la situación de emergencia sanitaria ha incidido positivamente en la mejora de la competencia digital de los docentes, ya que la transformación de la enseñanza presencial a los entornos virtuales ha ayudado a poner en práctica estrategias, recursos y habilidades docentes que no se ponen en práctica en la realidad cotidiana del aula. Si bien es verdad que son muchos los aspectos que no han sido trabajados con suficiente eficacia, como la atención a alumnos con necesidades educativas especiales.

El uso de la tecnología educativa y su inclusión en la sociedad actual como pieza vertebradora, ha provocado cambios en la enseñanza del profesorado, ha introducido nuevos recursos y metodologías emergentes en los centros educativos y los centros de enseñanza han de facilitar la alfabetización digital del alumnado (cuya trascendencia vaya más allá del entorno escolar) mediante la propia alfabetización digital del profesorado (Girón-Escudero, Cózar-Gutierrez y González-Calero, 2019), por ello es necesario desenvolverse correctamente a través de la capacitación a los docentes en su formación inicial y mediante la formación continua y permanente.

Esta necesidad de formación y desarrollo de la competencia digital en los docentes viene dada por un correcto uso de la tecnología (de manera eficaz y adecuado), que permita utilizarla en la dinámica de aula a través de su adaptación a los estudiantes y a los aprendizajes que estos han de conseguir.

La situación provocada por la COVID-19, ha puesto de manifiesto las carencias de nuestro sistema educativo en cuanto al correcto uso de las Tecnologías de la Información y la Comunicación, ya que la mejora educativa (que se busca desde los

Revista de Comunicación y Salud, 2020, Vol. 10, nº 2, pp. 483-502 
La competencia digital del profesorado y la atención a la diversidad durante la COVID-19

desarrollo normativos y legales, así como desde los centros educativos), ha de partir de un cambio de dirección de los modelos pedagógicos y se ha de ser un fin común de toda la comunidad educativa. Para ello, no se puede relegar a un segundo plano la inclusión de la tecnología en la dinámica general de los centros y tenerla en cuenta como elemento principal en el proceso de enseñanza y aprendizaje, ya que juega un papel destacado en la vida cotidiana del alumnado (el cual podemos considerar como nativo digital) y la escuela ha de ejercer su papel educativo a través de la enseñanza del correcto uso de las herramientas tecnológicas a través de su uso.

Para ello, la competencia digital docente supone un requisito imprescindible para alcanzar la excelencia en el ámbito educativo. Las TIC en el aula se han convertido en una herramienta cuya utilización está condicionada por el perfil formativo del docente (Pozo, López, Fernández y López, 2020). La COVID-19, nos ha ayudado a identificar los déficits en la actitud y formación tecnológica docente y ha puesto de relieve la necesidad de actualizar las prácticas educativas, ya que se ha demostrado que su ineficiente uso en los centros ( $\mathrm{y}$ la falta de adquisición de recursos tecnológicos para el alumnado, por parte de las administraciones competentes) favorece la brecha digital y la categorización del alumnado en aquellos cuyos tienes recursos y formación y aquellos no.

Esta premisa nos permite, de nuevo, destacar la necesidad de actualización docente y de que las herramientas tecnológicas de los centros educativos sean integradas en los corpus metodológicos y en las propuestas pedagógicas, donde el docente sea considerado como un guía y orientador del aprendizaje de la competencia digital del alumnado y pueda efectuarse una correcta inclusión de las tecnologías emergentes en los centros educativos (López, Pozo, Fuentes y Romero, 2019), ya que permiten fomentar y mejorar el aprendizaje significativo constructivista, la motivación y los resultados académicos.

Por otro lado, autores como Sánchez, Romero y Harari, citados por Sánchez (2019) afirman que las TIC favorecen el proceso de enseñanza y aprendizaje de los alumnos ACNEAES (Alumnado Con Necesidad Específica de Apoyo Educativo) considerándolo como un buen elemento curricular y metodológico que facilita el aprendizaje de los alumnos en general y, en particular, de aquellos que presentan necesidades educativas especiales.

Diversos estudios (Arnaiz, de Haro y Maldonado, 2019) han demostrado cómo las TIC pueden llegar a convertirse en un elemento decisivo para mejorar la calidad de vida de estas personas con algún tipo de discapacidad, siendo en algunos casos, una de las pocas opciones para poder acceder al currículum escolar, posibilitar la comunicación y facilitar su integración social. Por este motivo, se están implementando cada vez más estos recursos en los planes educativos, con el fin de dar una respuesta más individualizada a las necesidades propias de este colectivo (Easton, citado en Hernández y Sosa, 2018).

Ahora bien, no han de considerarse como una herramienta más, sino que deben formar parte de la programación curricular, lo que supone innovar en metodologías y 
La competencia digital del profesorado y la atención a la diversidad durante la COVID-19

estrategias didácticas. Para ello, deben buscarse tareas que, adaptadas a las características particulares de cada uno, permitan mejoras en su propio desarrollo y en el manejo de herramientas digitales. De esta manera, el proceso de enseñanzaaprendizaje en alumnos con alguna discapacidad se verá más alentado por el uso de estos medios digitales.

Son muchos los estudios que han apostado por la incorporación de las TIC en esta situación, considerándolas una herramienta de apoyo tanto para el docente como para el alumnado con NEE. Muchas son las páginas web 0 aplicaciones dirigidas especialmente a estos alumnos, garantizando que están sustentadas en las técnicas de usabilidad y accesibilidad. Usabilidad haciendo referencia a la capacidad que tiene un programa de ser comprendido, aprendido y usado por cualquier usuario en unas condiciones concretas; y accesibilidad refiriéndose al grado en el que todas las personas pueden visitar y utilizar páginas web y aplicaciones (Renilla, Pedrero y Sánchez, 2010).

¿Y por qué se deberían haber utilizado estos recursos como apoyo o refuerzo de los niños ACNEAES? En primer lugar, porque los medios digitales resultan ser muy atractivos y divertidos para quien los usa. Como si de una habilidad casi innata se tratase, este colectivo parece tener una afinidad natural con el uso de dispositivos tecnológicos. En segundo lugar, algunas investigaciones citadas en García, Garrote y Jiménez (2016) han señalado que las TIC ofrecen a este alumnado un entorno controlado, con situaciones predecibles e indicaciones perfectas y comprensibles. Además, darán la opción de repetir aquellas actividades o acciones que les resulten más placenteras las veces que ellos consideren oportunas; algo que resulta necesario para estos niños.

Por otro lado, podemos indicar que las TIC brillan por su adaptabilidad. En este sentido, se adecuan al proceso de enseñanza-aprendizaje no presencial, haciendo posible cualquier adaptación curricular que este alumnado precise, partiendo de las actividades propuestas al grupo-clase. Así mismo, se puede decir que las TIC se adaptan a las características de cada persona, favoreciendo ritmos y estilos de aprendizaje diferentes y otorgando una mayor individualización.

Siguiendo en la misma línea, hay estudios (Cabero y Ruiz-Palmero 2018) que destacan que, si bien las TIC permiten desarrollar aprendizajes autónomos, también ofrecen la posibilidad de realizar tareas de aprendizaje de manera conjunta a través de videoconferencias, chat, clases virtuales. Así, las TIC supondrían un medio para el desarrollo de habilidades emocionales y sociales, que harían posible la comunicación e interacción de estos con sus compañeros en la distancia (Corp, 2020).

Por ello, la educación y los centros escolares, tanto presenciales como on-line, deben ser un lugar de protección y reducción de las desigualdades sociales, donde los alumnos encuentran normalidad, estabilidad y esperanza (UNICEF, 2020). El sistema educativo debe ofrecer un sistema de protección presencial para el conjunto del alumnado, pero los principios inclusivos y las medidas de atención a la diversidad no 
pueden quedar relegados a un segundo plano en situaciones excepcionales de emergencia social (como la provocada por la COVID-19).

No queremos cerrar esta investigación sin exponer una reflexión personal a la que hemos llegado los investigadores: Quizás se ha perdido la oportunidad, durante estos meses de comunicación virtual, de explorar los miles de recursos, programas, juegos, métodos y buenas prácticas que pueden ayudar al docente en el acompañamiento didáctico de las potencialidades de alumnos con necesidades educativas especiales. Quizás se ha perdido la oportunidad de comprobar que los recursos TIC no sólo pueden ser un buen aliado en el proceso, sino un buen instrumento de empoderamiento de los alumnos. Quizás se ha perdido la oportunidad de cerrar el círculo de aprendizaje entre el alumno, el docente y la familia apoyado por un dispositivo tecnológico. Pero...aún estamos a tiempo.

\section{REFERENCIAS}

Arnaiz, P., de Haro, R., Maldonado, R.M. (2019). Barriers to Student Learning and Participation in an Inclusive School as Perceived by Future Education Professionals. Journal of New Approaches in Educational Research, 8(1),18-24. doi: $\underline{10.7821 / \text { naer.2019.1.321 }}$

Bisquerra, R. (2009). Metodología de la Investigación Educativa. Madrid: Muralla.

BOE. (2006, 4 de mayo). Ley Orgánica 2/2006, de 3 de mayo, de Educación. Boletín Oficial del Estado. Recuperado de: https://tinyurl.com/nfa4h2v

BOE. (2013, 10 de diciembre). Ley Orgánica 8/2013, de 9 de diciembre, para la mejora de la calidad educativa. Boletín Oficial del Estado. Recuperado de: https://tinyurl.com/y5gpwlf7

BOE. (2020a, 14 de marzo). Real Decreto 463/2020, de 14 de marzo, por el que se declara el estado de alarma para la gestión de la situación de crisis sanitaria ocasionada por el COVID-19. Boletín Oficial del Estado. Recuperado de: https://tinyurl.com/y59g72pg

BOE. (2020b, 22 de abril). Orden EFP/365/2020, de 22 de abril, por la que se establecen el marco y las directrices de actuación para el tercer trimestre del curso 2019-2020 y el inicio del curso 2020-2021, ante la situación de crisis ocasionada por el COVID-19. Boletín Oficial del Estado. Recuperado de: https://tinyurl.com/y4jnllxo

Cabero, J., y Ruiz-Palmero, J. (2018). Las Tecnologías de la información y la comunicación para la inclusión: reformulando la brecha digital. International Journal of Educational Research and Innovation, 9, 16-30. Recuperado de: https://tinyurl.com/y6xrnu5d

Corps, L.M. (2020). Las tecnologías de la información y comunicación en el proceso de enseñanza y aprendizaje del alumnado con trastorno del espectro autista. Universidad de Castilla-La Mancha 
La competencia digital del profesorado y la atención a la diversidad durante la COVID-19

Cueto García, C. (2020). El COVID19 acelera la digitalización de los profesores. Computerworld. Recuperado de: https://tinyurl.com/y6svev7r

Diez-Gutierrez, E. \& Gajardo-Espinoza, K. (2020). Educar y Evaluar en Tiempos de Coronavirus: la Situación en España. Multidisciplinary Journal of Educational Research, 10(2), 102-134. doi: 10.17583/remie.2020.5604

EFE. (2020). El Gobierno recomienda el cierre de los colegios en todas las comunidades. Agencia EFE. Recuperado de: https://tinyurl.com/y628ggyl

Flick, U. (2012). Introducción a la Investigación Cualitativa. Madrid: Ediciones Morata S. L.

Fuentes, A. y López, J. (2018). TIC-TAC: Las tecnologías de la información y la comunicación en la era del acceso. Una experiencia en la educación superior. En del I. Arco y P. Silva (Eds.), Tendencias nacionales e internacionales en organización educativa: Entre la estabilidad y el cambio (pp. 712-727). Madrid: Wolters Kluwer.

Gabarda Méndez, V., Rodríguez Martín, A., y Moreno Rodríguez, M.D. (2017). La competencia digital en estudiantes de magisterio. Análisis competencias y percepción personal del futuro maestro. Educatio Siglo XXI, 2(35), 253-274. doi: $\underline{10.6018 / \mathrm{j} / 298601}$

García, S., Garrote, D., y Jiménez, S. (2016). Uso de las TIC en el Trastorno de Espectro Autista: aplicaciones. EDMETIC, Revista de Educación Mediática y TIC, 5(2), 134-157. doi: $10.21071 /$ edmetic.v5i2.5780

Girón-Escudero, V., Cózar-Gutierrez, R. y González-Calero Somoza, J.A. (2019). Análisis de la autopercepción sobre el nivel de competencia digital docente en la formación inicial de maestros/as. Revista Electrónica Interuniversitaria de Formación del Profesorado, 22(3), 193-2018. doi: 10.6018/reifop.373421

Hernández Vásquez, M., \& Sosa Hernández, M. (2018). Uso de Tecnologías de Información y Comunicación (TIC) en inclusión escolar de estudiantes con Trastornos del Espectro Autista (TEA). Contextos: Estudios De Humanidades Y Ciencias Sociales, (41). Recuperado de: https://tinyurl.com/y49a3ces

Hernando Ramírez Atehortúa, F. y Zwerg-Villegas, A.M. (2012). Metodología de la investigación: más que una receta. $A D$-Minister, 20 (enero-junio), 91-111. Recuperado de: https://tinyurl.com/yyqubk6z

Juste, M. (2020). Coronavirus: Grandes y pequeñas empresas ofrecen gratis sus plataformas de educación online. Expansión. Recuperado de: https://tinyurl.com/y4n3v8oj

López Belmonte, J., Pozo Sánchez, S., Fuentes Cabrera, A., y Romero Rodríguez, J.M. (2019). Análisis del liderazgo electrónico y de la competencia digital del profesorado de cooperativas educativas de Andalucía (España). Multidisciplinary Journal of Educational Research, 9(2), 194-223. doi: 10.17583/remie.2019.4149 
La competencia digital del profesorado y la atención a la diversidad durante la COVID-19

Marqués, P. (2008). Las competencias digitales de los docentes. Recuperado de: https://tinyurl.com/yxaysrcz

Martín, J.M., \& Rogero, J. (2020). El coronavirus y la asfixia educativa: el confinamiento deja sin protección a la infancia más vulnerable. eldiario.es. Recuperado de: https://tinyurl.com/yyvbak9u

Moreno, S.M. (2020). La innovación educativa en los tiempos del coronavrus. Salutem Scientia Spiritus, 6(1), 14-26. Recuperado de: https://tinyurl.com/y2bu99jw

Moreno Rodríguez, M.D., Gabarda Méndez, V. y Rodríguez Martín, A. M. (2018). Alfabetización informacional y competencia digital en estudiantes de magisterio. Profesorado. Revista de Currículum y Formación de Profesorado, 22(3), 253-270. doi: $10.30827 /$ profesorado.v22i3.8001

Pozo Sánchez, S., López Belmonte, J., Fernández Cruz, M. \& López Núñez, J.A. (2020). Análisis correlacional de los factores incidentes en el nivel de competencia digital del profesorado. Revista Electrónica Interuniversitaria de Formación del Profesorado, 23(1), 143-159. doi: 10.6018/reifop.396741

Renilla, M.R., Pedrero, A., y Sánchez, A. (2010). Autismo y TIC's. International Journal of Developmental and Educational Psychology, 4(1), 169-177. Recuperado de: https://tinyurl.com/y3lyytvg

Roig R. \& Carolina Flores, C. Conocimiento tecnológico, pedagógico y disciplinario del profesorado: el caso de un centro educativo inteligente. EDUTEC, Revista Electrónica de Tecnología Educativa, 47. Recuperado de https://tinyurl.com/y6x4vvy7

Sánchez, T. (2019). Programa de buenas prácticas TIC en personas con Trastorno del Espectro Autista. Reflexión e Investigación Educacional, 2(1), 85-92. Recuperado de: https://tinyurl.com/y43bc4th

Trujillo, F. (2020). ¿Evaluación del curso? Escenarios posibles para el futuro inminente de la educación. eldiariodelaeducacion.com. Recuperado de: https://tinyurl.com/y2ey9rl6

UNICEF (2020). La educación frente al COVID-19. Propuestas para impulsar el derecho a la educación durante la emergencia. Recuperado de: https://tinyurl.com/y48t3wu6

Valle, J y Manso, J. (20013). Competencias clave como tendencia de la política educativa supranacional de la Unión Europea. Revista de Educación, Extraordinario, 12-23. doi: $\underline{10.4438 / 1988-592 X-R E-2013-E X T-255}$ 
La competencia digital del profesorado y la atención a la diversidad durante la COVID-19

\section{AUTORES}

\section{Óscar Gómez Jiménez}

Profesor de Educación Primaria en la especialidad de Inglés, Pedagogía Terapéutica y Audición y Lenguaje en el Colegio Sagrado Corazón de Jesús y María Inmaculada (Miajadas, Cáceres), master en Psicopedagogía y doctorando en Investigación en Humanidades, Artes y Educación por la Universidad de Castilla-La Mancha. Especialista en atención a la diversidad y sus líneas de investigación son la legislación educativa, la atención a la diversidad y la educación inclusiva.

Orcid ID: http://orcid.org/0000-0002-6581-4481

Google Scholar: https://scholar.google.com/citations?hl=es\&user=BtVNhnAAAAAJ

ResearchID: https://publons.com/researcher/3846085/oscar-gomez-jimenez/

\section{Javier Rodríguez Torres}

Doctor en Pedagogía por la Universidad de Alcalá (España) en la línea de formación del profesorado y TIC. Además de maestro de Educación Infantil y Primaria con varias especialidades, realizó Máster de Psicología Escolar en la Universidad Complutense de Madrid. Miembro del Grupo de Investigación CIBERIMAGINARIO-UCLM. En la actualidad es Director Académico de Economía y Planificación de la Universidad de Castilla - La Mancha, también es coordinador del Máster de Secundaria en el Campus de Toledo y director del postgrado de Discapacidad, Inclusión y Ciudadanía. Nuevos Enfoque. Las líneas de investigación, con respecto a las TIC, son su integración curricular, las diferencias de género en su inclusión y currículo, los discursos de poder saber que se generan en el uso y aplicación de las tecnologías.

Orcid ID: http://orcid.org/0000-0003-1029-5562

Google Scholar: https://scholar.google.es/citations?user=CM63 cAAAAAJ\&hl=es

ResearchID: https://publons.com/researcher/G-3899-2015/

\section{Purificación Cruz Cruz}

Doctora en Psicopedagogía. Especialista Educación Infantil y familiar. Profesora de la Facultad de Educación de la Universidad de Castilla la Mancha, Toledo. Maestra de Educación Infantil desde hace más de 30 años en el colegio Nuestra Señora de los Infantes. Especialista en programación, creatividad y gestión educativa. Conferenciante en distintas asociaciones de padres y colectivos de maestros. Autora de distintos libros y artículos científicos sobre educación e inteligencia emocional. Conferenciante en la Universidad de Zacatecas (México) y en la Universidad del Norte (Colombia). Ponente en numerosos congresos nacionales e internacionales sobre educación. Autora de numerosos libros y artículos científicos. Experta en inteligencia emocional.

Orcid ID: https://orcid.org/0000-0001-5637-3007

Google Scholar: https://scholar.google.es/citations?user=Oiu7qhoAAAAJ\&hl=es

ResearchID: https://www.researchgate.net/profile/Purificacion Cruz 